\title{
THE CONFIGURATION, CONFORMATION AND DEGRADATION OF POLYVINYL CHLORIDE
}

\author{
F. A. BoveY
}

Bell Telephone Laboratories Incorporated, Murray Hill, New Jersey 07974 $U S A$

\begin{abstract}
The proton spectrum of poly(vinyl chloride) prepared by free-radical polymerization has been studied at $220 \mathrm{MHz}$ and interpreted in terms of pentads for the $\alpha$-protons and tetrads for the $\beta$-protons. The chemical shifts are in good agreement with those reported elsewhere using selectively deuterated polymers. The magnitudes of the vicinal coupling constants indicate that in the solvents employed, the isotactic dyads are in approximate equilibrium between the conformations which, if repeated, result in a $3_{1}$ helix, whereas syndiotactic dyads are probably in the conformation with the backbone in a planar zigzag structure. The tetrad and pentad relative intensities are consistent with Bernoullian chain propagation statistics, with a value $P_{m}$ (the probability of isotactic placement) of 0.43 .

Radiolysis of poly(vinyl chloride) (PVC) was studied by ultraviolet and visible spectroscopy following post-irradiation storage at selected temperatures in a cryostatic cell. A sequence of alkyl, allyl and polyenyl radicals followed by chain transfer to PVC leads to polyene formation. Allyl, dienyl and trienyl radicals were associated with absorption bands at 2520, 2910, and $3300 \AA$, respectively. Radical intermediates react readily with oxygen.
\end{abstract}

\section{INTRODUCTION}

In this paper, I wish to report on recent work carried out at the Bell Telephone Laboratories on the structure and chemical reactivity of polyvinyl chloride. Although many techniques and types of spectroscopy have been applied to this polymer in this and other laboratories, our principal tools in this work have been high resolution $\mathrm{nmr}$ for the study of its configuration and conformation and esr and uv spectroscopy for the study of its chemical behaviour, in particular the dehydrochlorination reaction which is initiated by high energy radiation.

\section{STEREOCHEMICAL CONFIGURATION $\dagger$}

Interpretation of the $\mathrm{nmr}$ data for polyvinyl chloride has been protracted and controversial, in part because of ambiguities in the spectra and in part

$\dagger$ In the following discussion, familiarity with the basic concepts used in interpreting the nmr spectra of vinyl polymers will be assumed. For those needing it, a review of these concepts can be found in Acc. Chem. Res. 1, 175 (1968). 


\section{F. A. BOVEY}

because of conflicting conclusions drawn from other measurements, particularly from vibrational spectroscopy (infrared and Raman). The ambiguities and disagreements appear to have been largely resolved by $\mathrm{nmr}$ observations at higher magnetic fields. Study of model 2,4-dichloropentanes and 2,4,6trichloroheptanes has been very helpful in deducing the conformation of the polymer chain, but at one time caused some confusion with regard to its stereochemical configuration. This concerned mainly the $\beta$-methylene spectrum, in which the chemical shift difference between the protons decreases with the number of neighbouring $m$ dyads. We shall not discuss here all the contributions to this problem, which have been extensive ${ }^{1-24}$, but will instead emphasize our most recent results.

One would expect on the simplest basis that the $\alpha$-proton spectrum would be a pentuplet from coupling to the four $\beta$-protons, and the $\beta$-protons a triplet from coupling to the two $\alpha$-protons. In fact, this is not observed even at $60 \mathrm{MHz}$. Johnsen ${ }^{8}$ reported that at this frequency the $\alpha$-proton spectrum of polyvinyl chloride in chlorobenzene at $160^{\circ}$ gave an apparent 'pentuplet' at $5.53 \tau$, as expected, but that the $\beta$-protons appeared as five peaks near $8.8 \tau$. These were interpreted as two overlapping triplets centred at $7.78 \tau$

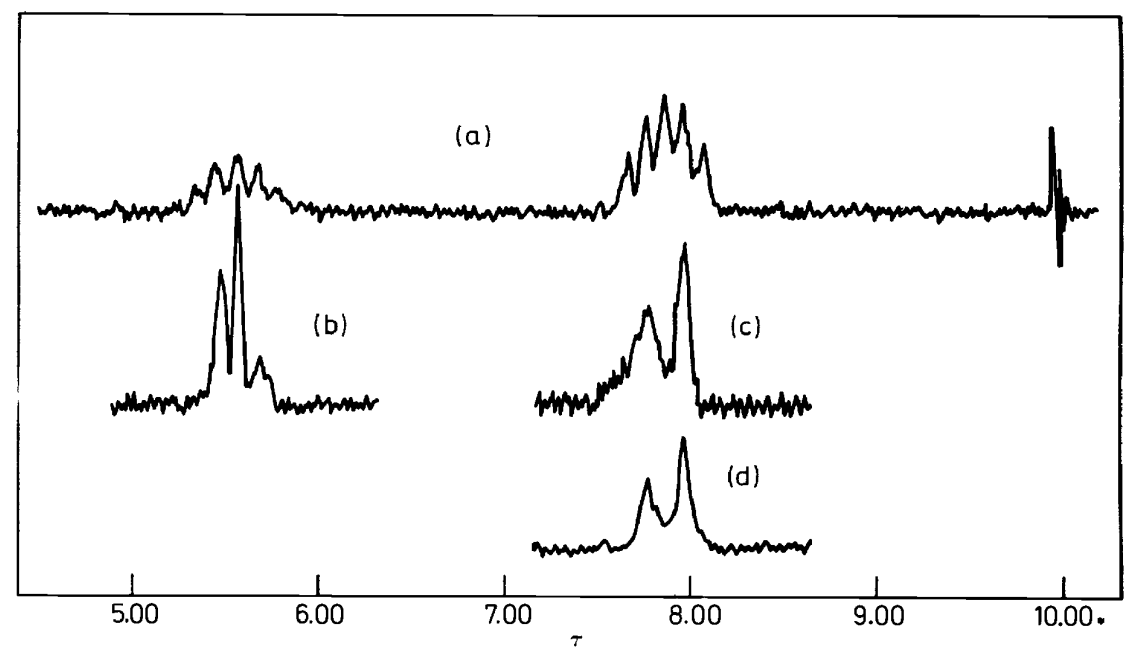

Figure 1. Normal and decoupled spectra of polyvinyl chloride, observed in 15 per cent $(\mathrm{w} / \mathrm{v})$ solution in chlorobenzene. (a) normal spectrum (at $170^{\circ}$ ); (b) $\alpha$-protons upon irradiation of $\beta$-protons $\left(150^{\circ}\right)$; (c) $\beta$-protons upon irradiation of $\alpha$-protons $\left(150^{\circ}\right)$; (d) poly- $\alpha$ - $\mathrm{d}_{1}$-vinyl chloride $\left(156^{\circ}\right)$ (reference 12$)$.

and $7.96 \tau$, corresponding to meso and racemic methylene groups, respectively. This interpretation was questioned ${ }^{1,11,14}$ because it interpreted the meso methylene groups as homosteric, i.e., as having magnetically equivalent protons within experimental error, in contradiction to basic principles. However, it received confirmation from double resonance experiments ${ }^{12,13}$ as shown in Figure 1. (The polymers illustrated were prepared with a free 
radical initiator at $50^{\circ}$ ). The normal spectrum is shown in (a). When the $\beta$-protons are decoupled from the $\alpha$-protons two peaks are observed, separated by $0.20 \mathrm{ppm}$. (c) A very similar spectrum is shown by the polymer of $\alpha$-deuterovinyl chloride CIDC $=\mathrm{CH}_{2}$ (spectrum d). When the $\beta$-protons are irradiated the $\alpha$-proton resonance shows peaks at $\tau 5.48,5.59$ and 5.71 (spectrum b). The dependence of the decoupled $\alpha$-proton spectrum upon the decoupling difference frequency $\Delta v$ can be employed to demonstrate that these peaks correspond to $r r, m r$, and $m m$ triads, respectively, assuming the correctness of the dyad assignments, which are compatible with model compound measurements. Relative peak areas in the $\alpha$-and $\beta$-proton spectra indicated the polymer to be nearly atactic with a small preponderance of $r$ sequences.

Careful examination ${ }^{21}$ of spectrum (d) in Figure 1 shows that a dyad analysis is inadequate, because there are small peaks which cannot be

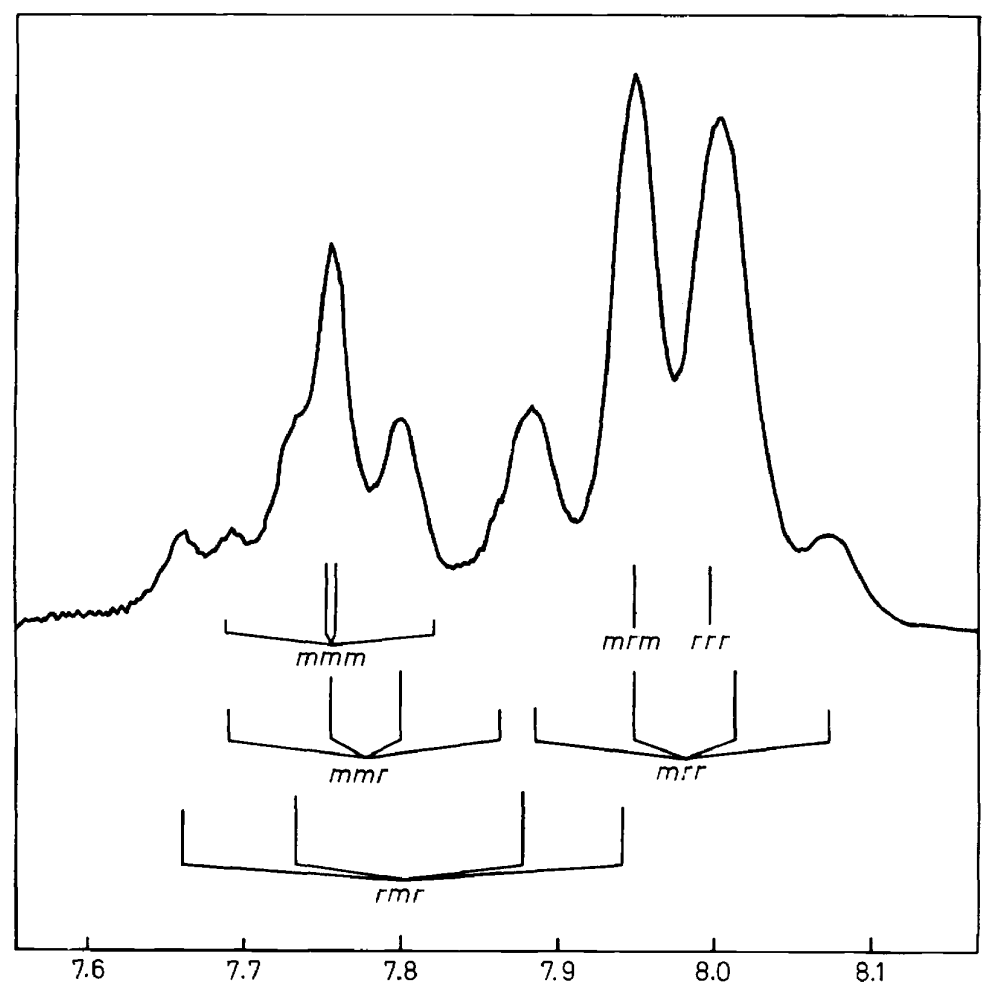

Figure 2. $220 \mathrm{MHz}$ proton spectrum of poly- $\alpha-\mathrm{d}_{1}$-vinyl chloride, observed in 15 per cent $(\mathrm{w} / \mathrm{v})$ solution in chlorobenzene at $140^{\circ}$ (reference 23 ).

accounted for in this way. Yoshino and Komiyama ${ }^{15}$ supplied the explanation for these by showing that in the $100 \mathrm{MHz}$ spectrum of poly- $\alpha, \beta-\mathrm{d}_{2}$-vinyl chloride, peaks corresponding to all ten chemical shifts of the six $\beta$-methylene tetrads can be distinguished. Such discrimination is easier in the $\alpha, \beta-d_{2}$ 


\section{F. A. BOVEY}

polymer than in the $\alpha-d_{1}$ polymer because in the latter the heterosteric tetrad resonances ( $m m m, m m r, r m r$, and $m r r$ ) appear as $\mathrm{AB}$ quartets, with the resonance intensity concentrated at the centre, rather than as more widely spaced pairs of singlets.

The $220 \mathrm{MHz}$ spectrum of poly- $\alpha-\mathrm{d}_{1}$-vinyl chloride has been shown ${ }^{23}$ to be consistent with the chemical shift assignments of Yoshino and Komiyama ${ }^{15}$. At these higher frequencies, many more features can be seen (Figure 2); the chance of fitting the spectrum with a self-consistent but erroneous set of assignments becomes very small. (Figure 2 should be compared with Figure 1-d, the same polymer at $60 \mathrm{MHz}$.) The analysis of the spectrum into four $\mathrm{AB}$ quartets $\left(J_{\mathrm{AB}}=-15 \mathrm{~Hz}\right)$ for the heterosteric tetrads

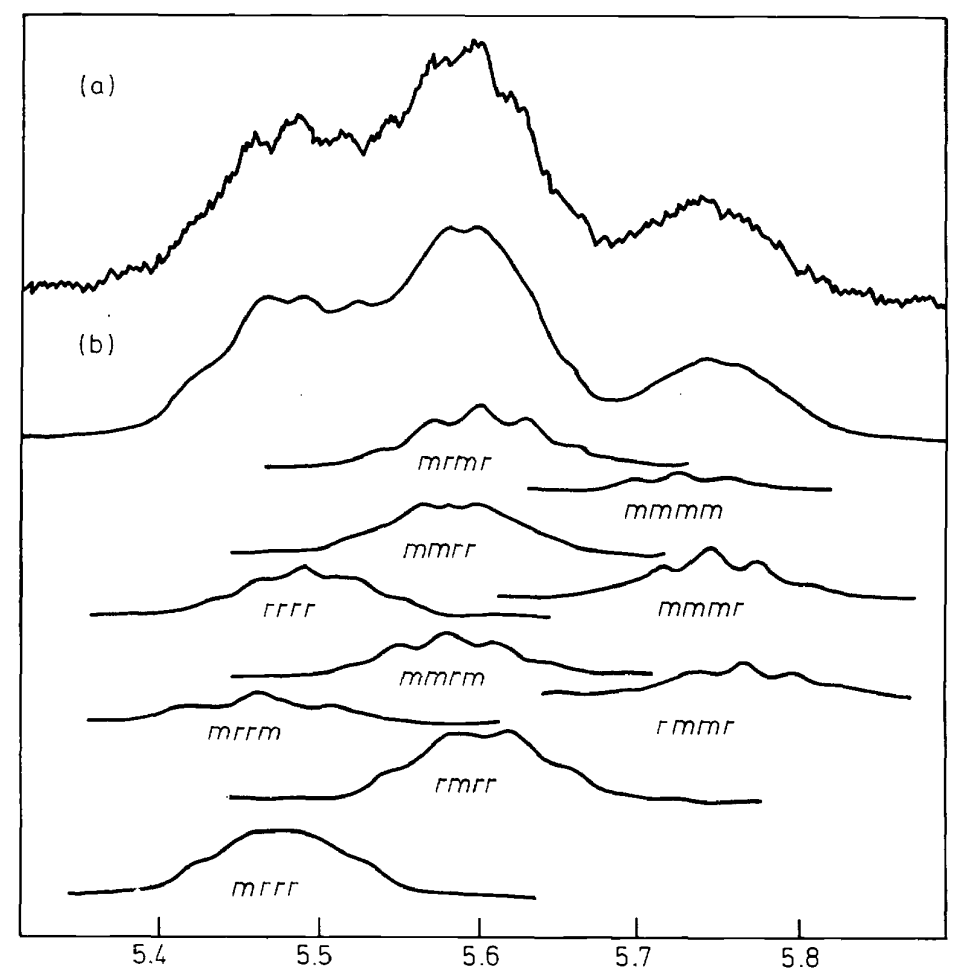

Figure 3. (a) $220 \mathrm{MHz} \alpha$-proton spectrum of polyvinyl chloride, observed in 5 per cent solution in chlorobenzene at $140^{\circ}$; (b) spectrum calculated using the parameters in Table 1, with the component pentad subspectra (reference 23).

and two singlets for the homosteric tetrads is indicated. Their intensities correspond to Bernoullian statistics with a probability of isotactic placement, $P_{m}$, of 0.43 . Comparing the chemical shifts in the series $m m m, m m r, r m r$, it is seen that the configuration of the second nearest asymmetric centre has as large an effect on the differentiation of the chemical shifts in each methylene group as the configuration of the adjacent $\alpha$-carbons. The fact that the 


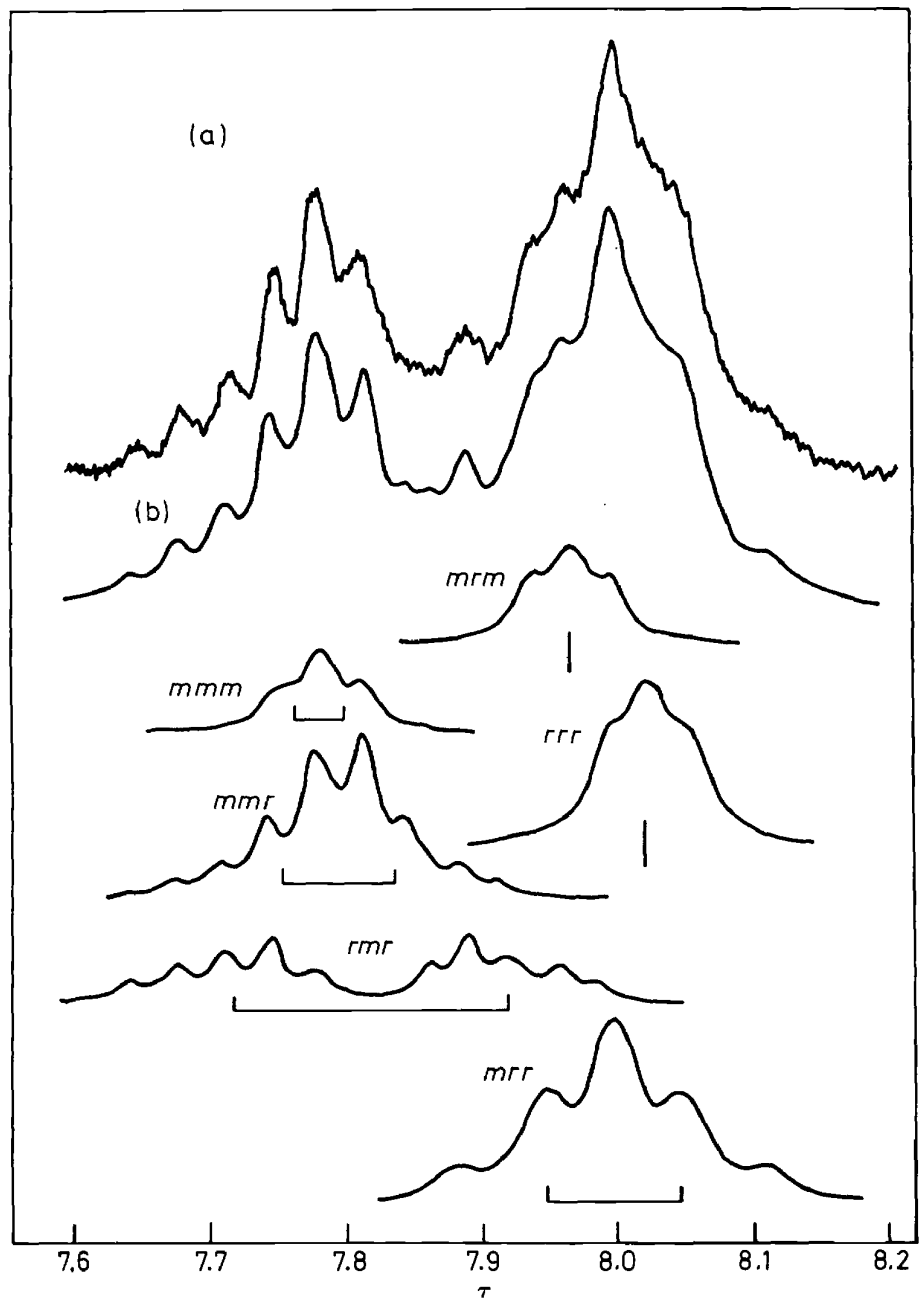

Figure 4. (a) $220 \mathrm{MHz} \beta$-proton spectrum of polyvinyl chloride, observed in 5 per cent solution in chlorobenzene at $140^{\circ}$; (b) spectrum calculated using the parameters in Table 1 , with the component tetrad subspectra (reference 23).

least heterosteric character is found in the $\mathrm{mmm}$ tetrad indicates that chemical shift differentiation from the nearest and next nearest $\alpha$-carbons of the same configuration must be similar in magnitude but opposite in sign. There is thus a much less rapid attentuation of the influence of the $\alpha$-carbon configuration along the chain than in polymethyl methacrylate.

A second consistent trend is the upfield movement of the mean tetrad chemical shift when neighbouring $m$ dyads are replaced by $r$. These chemical shift correlations are no doubt intimately related to chain conformation as well as configuration. At present, no consistent effort has been made to understand them in polar chains such as these. It is probable that the present 


\section{F. A, BOVEY}

theoretical framework for the interpretation and prediction of chemical shifts is inadequate to the task, at least in a semiempirical fashion.

The undeuterated polymer spectrum has also yielded to a more complete analysis. Johnsen and Kolbe ${ }^{22}$ have employed decoupling to distinguish pentad resonances in the $\alpha$-proton spectrum at $100 \mathrm{MHz}$. Cavalli ${ }^{24}$ et al. obtained very similar results for poly- $\beta-\mathrm{d}_{2}$-vinyl chloride at $100 \mathrm{MHz}$ and even at $60 \mathrm{MHz}$. Using pentad chemical shifts established by these authors and the tetrad chemical shifts just discussed, Heatley et al. ${ }^{23}$ were able to carry out a computer simulation of the entire polyvinyl chloride spectrum. Figure 3 shows the $\alpha$-proton spectrum and Figure 4 the $\beta$-proton spectrum, as observed at $220 \mathrm{MHz}$ in chlorobenzene at $140^{\circ}$. Spectrum (b) in each figure is the simulated spectrum. Below these are the calculated pentad (Figure 3) and tetrad (Figure 4) subspectra. The pentad subspectra each represent a single type of proton, the resonance of which is split by coupling to the $\beta$-proton. The tetrad spectra correspond to the same chemical shifts (indicated by vertical bars, which are connected by horizontal bars for the heterosteric methylenes) shown in Figure 2, but now complicated by vicinal couplings. These subspectra are those which would be expected for polymers each of which contained only the repeating sequence in question. The subspectra are summed by the computer assuming the same Bernoullian intensities $\left(P_{m}=0.43\right)$ as in Figure 2 . The spectra in three solvents ( $o$-dichlorobenzene and pentachloroethane in addition to chlorobenzene) are calculated on the assumption that the polymer chain may be satisfactorily represented as a cyclic 'dimer', a model originally suggested by Tincher ${ }^{11,14}$ and successfully used for the computer simulation of many polymer spectra. Agreement within experimental error (there is clearly more leeway in Figure 3 than in Figure 4) is obtained using the parameters shown in Table 1; chemical shifts of tetrad and pentad protons are expressed in $\mathrm{Hz}$ with respect to $\mathrm{mrm}$ as zero in each solvent (actual $\tau$-values given for $\mathrm{mrm}$ in each solvent):

Table 1. Chemical shifts (see text) and $J$-couplings (Hz) for polyvinyl chloride (220 MHz)

\begin{tabular}{|c|c|c|c|c|c|}
\hline \multicolumn{2}{|r|}{ 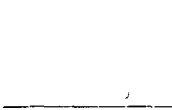 } & $\begin{array}{l}\text { Chloro- } \\
\text { benzene }\end{array}$ & $\begin{array}{l}\text { Solvent- } \\
\text { o-Dichloro- } \\
\text { benzene }\end{array}$ & $\begin{array}{l}\text { Pentachloro- } \\
\text { ethane }\end{array}$ & $\begin{array}{l}\text { Relative } \\
\text { intensity }\end{array}$ \\
\hline \multicolumn{6}{|c|}{$m$ centred tetrads } \\
\hline \multirow[t]{2}{*}{$\mathrm{mmm}$} & $v_{\mathbf{A}}$ & -44.5 & -40.5 & -48.5 & 0.080 \\
\hline & $v_{\mathrm{B}}$ & -46.0 & -32.5 & -42.5 & \\
\hline \multirow[t]{2}{*}{$m r m$} & $v_{\mathrm{A}}$ & -46.0 & -42.5 & -48.5 & 0.208 \\
\hline & $v_{\mathrm{B}}$ & -28.5 & -28.5 & -32.5 & \\
\hline \multirow[t]{2}{*}{$r m r$} & $v_{\mathrm{A}}$ & -55.0 & -49.0 & -59.5 & 0.141 \\
\hline & $v_{\mathrm{B}}$ & -10.5 & -10.5 & -16.5 & \\
\hline \multicolumn{2}{|l|}{$J_{\mathrm{AB}}$} & -15.0 & -15.0 & -15.0 & \\
\hline \multicolumn{2}{|l|}{$J_{\mathrm{AX}}$} & 7.5 & 7.5 & 7.5 & \\
\hline \multicolumn{2}{|l|}{$J_{\mathbf{B X}}^{\mathrm{AX}}$} & 6.5 & 6.5 & 6.5 & \\
\hline \multicolumn{2}{|c|}{ Line width } & 5.0 & 5.0 & 5.0 & \\
\hline \multicolumn{6}{|c|}{$r$ centred tetrads } \\
\hline \multicolumn{2}{|l|}{$m r m$} & 0 & 0 & 0 & 0.105 \\
\hline \multicolumn{2}{|l|}{$m r m \tau$} & 7.96 & 7.90 & 7.87 & \\
\hline
\end{tabular}




\begin{tabular}{|c|c|c|c|c|}
\hline & \multicolumn{3}{|c|}{-Solvent-_ } & \multirow[b]{2}{*}{$\begin{array}{l}\text { Relative } \\
\text { intensity }\end{array}$} \\
\hline & $\begin{array}{l}\text { Chloro- } \\
\text { benzene }\end{array}$ & $\begin{array}{l}o \text {-Dichloro- } \\
\text { benzene }\end{array}$ & $\begin{array}{l}\text { Pentachloro- } \\
\text { ethane }\end{array}$ & \\
\hline$m r r v_{\mathbf{A}}$ & -4.0 & -3.5 & -4.5 & 0.281 \\
\hline$v_{\mathrm{B}}$ & 17.5 & 17.0 & 9.5 & \\
\hline$r r r$ & 12.5 & 12.5 & 1.0 & 0.185 \\
\hline$J_{\mathbf{A A}}$ & -15 & -15 & -15 & \\
\hline$J_{\mathrm{AX}}$ & 11.0 & 11.0 & 10.0 & \\
\hline$J_{\mathrm{AX}}$ & 2.0 & 2.0 & 3.0 & \\
\hline Line width & 7.0 & 6.0 & 5.0 & \\
\hline \multicolumn{5}{|l|}{ Pentads } \\
\hline mrrm & -543 & & -527 & 0.060 \\
\hline$m r r r$ & -541 & & -525 & 0.160 \\
\hline$r r r r$ & -537 & & -526 & 0.105 \\
\hline $\mathrm{mmrm}$ & -517 & & -513.5 & 0.091 \\
\hline$m m r r$ & -517 & & -513.5 & 0.119 \\
\hline$m r m r$ & -513 & & -507.5 & 0.119 \\
\hline$r m r r$ & -513 & & -505.5 & 0.160 \\
\hline $\mathrm{mmmm}$ & -485 & & -492 & 0.035 \\
\hline$r m m r$ & -477 & & -479 & 0.060 \\
\hline Line width & 5.0 & & 5.0 & \\
\hline
\end{tabular}

The interpretation of the vicinal couplings will be discussed in the next section.

Let us now briefly consider the effect of temperature and solvent on the stereoregularity of free radical vinyl chloride polymers, which has been a matter of some dispute. It is well known ${ }^{25}$ that for Bernoullian propagation the differences in activation enthalpies and entropies for isotactic and syndiotactic propagation (the measurable quantities) are given by:

$$
\Delta H_{m}^{\ddagger}-\Delta H_{r}^{\ddagger}=\Delta\left(\Delta H_{p}^{\ddagger}\right)=-R \frac{\partial \ln \left[P_{m} /\left(1-P_{m}\right]\right.}{\partial(1 / T)}
$$

and

$$
\Delta S_{m}^{\ddagger}-\Delta S_{r}^{\ddagger}=\Delta\left(\Delta S_{p}^{\ddagger}\right)=R \ln \left[P_{m} /\left(1-P_{m}\right)\right]+\Delta\left(\Delta H_{p}^{\ddagger}\right) / T
$$

It is also possible in principle to deduce these quantities from the dependence of the infrared spectra on the temperature of polymerization.

Fordham et al. ${ }^{26}$ estimated a value of 600 cal for $\Delta\left(\Delta H_{p}^{\ddagger}\right)$, while Bovey and Tiers ${ }^{9}$ suggested a value of only ca. $200 \mathrm{cal}$. Neither group had a substantial experimental basis for their conclusions, and it now appears that they may bracket the correct value. Germar et al. ${ }^{27 a}$ reported $\mathrm{nmr}$ and infrared data pointing to a much larger value of $\Delta\left(\Delta H_{p}^{\ddagger}\right)$ than either of these earlier estimates, but there were discrepancies in samples prepared at the lower temperatures. Bargon et al. ${ }^{18}$ reported $350 \mathrm{cal}$ and $1200 \mathrm{cal}$ for polymers prepared in emulsion systems with water and methanol respectively, as the continuous phase. Such a difference is rather hard to rationalize. Bovey et al. ${ }^{21}$ deduced a value of $310 \mathrm{cal}$ for solution-polymerized poly- $\alpha-\mathrm{d}_{1}$-vinyl chloride, using computer simulation of $60 \mathrm{MHz}$ spectra, and essentially the same chemical 


\section{F. A. BOVEY}

shift analysis just presented. Cavalli et al. ${ }^{24}$ reported 630 cal for bulk-polymerized polymer. In most studies positive values of $\Delta\left(\Delta S_{p}^{\ddagger}\right)$ of the order of 1 eu were obtained. It is not clear whether the reported differences in activation enthalpy are true effects.

There is, however, no doubt that polyvinyl chloride prepared at low temperature differs in its physical properties, particularly in crystallizability, from that prepared at normal temperatures ${ }^{16,26}$. Talamini and Vidotto ${ }^{27 b}$ suggested that $r$ blocks of four or five units might form crystallites, but in a Bernoullian structure with a $P_{m}$ of ca. 0.4 such blocks would constitute at most only ca. $0.1-0.2$ of the monomer units. There is now ample evidence that vinyl polymer chains may crystallize despite a high degree of configurational irregularity. It appears likely that the enhanced crystallizability is due primarily to decreased branching in the low-temperature polymers ${ }^{16,24,28} 30$ rather than to the relatively small increase in syndiotactic sequences (e.g., $P_{m}$ of 0.46 and 0.37 for polymers prepared at $100^{\circ}$ and $-78^{\circ}$, respectively ${ }^{21}$ ).

Rosen et $a .^{31}$ concluded from x-ray diffraction studies that polyvinyl chloride prepared by free radical initiation in solvents such as butyraldehyde is more stereoregular (presumably more syndiotactic) than when prepared in emulsion or in ketones. A number of similar claims have been made by other authors (references given in reference 16) for polyvinyl chloride and other vinyl monomers. However, Bovey and Tiers ${ }^{9}$ were not able to detect any difference in the stereoregularity of polyvinyl chloride so prepared, and this finding was confirmed by Böckman ${ }^{16}$, who suggested that decreased molecular weight led to the enhanced crystallinity reported.

\section{CHAIN CONFORMATION}

The interpretation of vinyl polymer $\mathrm{nmr}$ spectra in terms of chain conformation depends primarily on an understanding of the vicinal $J$ couplings, which of course are strongly dependent on the dihedral angle in the $\mathrm{H}-\mathrm{C}-\mathrm{C}-\mathrm{H}$ fragment. They are weighted averages over the allowed conformations, since rotation about $\mathrm{C}-\mathrm{C}$ bonds is fast on the $\mathrm{nmr}$ time scale under the conditions employed for polymer studies. Interpretation is made less precise by this fact and also by the relatively great line width (see Table 1) characteristic of polymer spectra. Study of the nmr (and infrared) spectra of small-molecule analogues, the 2,4-disubstituted pentanes and 2,4,6-trisubstituted heptanes, the spectra of which are better resolved, has greatly aided this interpretation. The Czechoslovak workers have made very important contributions to this field. I shall not discuss this work in detail here $\dagger$, but will employ the principal conclusions drawn from it. These are embodied in Figure 5, in which (a) represents the allowed conformations of a meso (i.e. isotactic) dyad, which interconverts rapidly between equi-energy mirror-image conformers:

$$
t g \rightleftharpoons g t
$$

When repeated, each conformation generates the $3_{1}$ helix commonly found for crystalline isotactic polymers. The high resolution spectra of a number of

$\dagger$ For an extended discussion see F. A. Bovey, Polymer Conformation and Configuration, Academic Press, New York, 1969. 
isotactic polymers have now been shown to be consistent with a strong predominance of this conformation, although minor proportions of others are not necessarily excluded ${ }^{32}$.

For $r$ dyads, the $t t$ and $g g$ conformations ((b) in Figure 5) are allowed. These too are in rapid equilibration but are not necessarily equivalent in energy. Two vicinal couplings, $J_{\mathrm{Ax}}$ and $J_{\mathrm{AX}}$, can be obtained from the $\mathrm{nmr}$

(a)

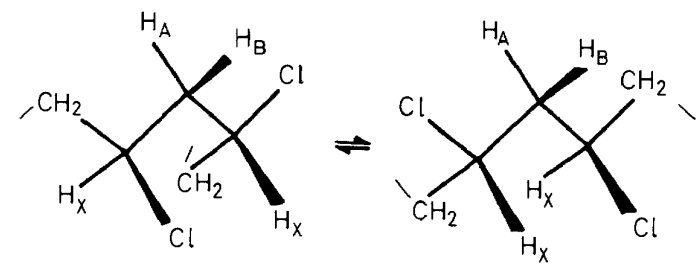

(tg)

(gt)

(b)

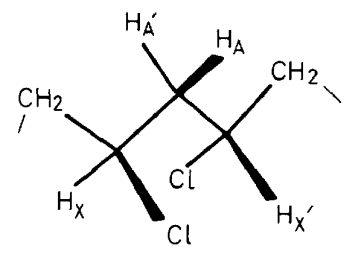

$(t+)$

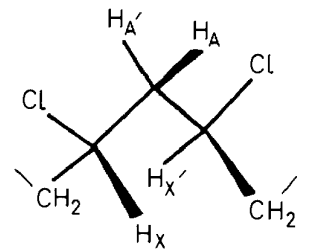

$(g g)$

Figure 5. Low energy conformations of (a) an $m$ dyad and (b) an $r$ dyad.

spectrum (see Table 1). Their values, averaged over the $t t$ and $g g$ conformations, are given by:

$$
\begin{aligned}
& J_{\mathrm{AX}}=J_{\mathrm{A}^{\prime} \mathbf{X}^{\prime}}=\mathrm{X}_{t t} J_{t}+\mathrm{X}_{g g} J_{g} \\
& J_{\mathrm{AX} \mathbf{X}^{\prime}}=J_{\mathbf{A}^{\prime} \mathbf{X}}=\mathrm{X}_{t t} J_{g}+\mathrm{X}_{g g^{\prime}} J_{t}
\end{aligned}
$$

where $\mathrm{X}_{t t(g g)}$ is the mole fraction of the $t t$ or $g g$ conformation. It is assumed that all coupling constants between trans protons take a value $J_{t}$ and all coupling constants between gauche protons take a value $J_{g}$. Such an approximation is sufficient for the purpose of this discussion. Since it,is-known that $J_{t} \sim 11-13 \mathrm{~Hz}$ and $J_{g} \sim 1-3 \mathrm{~Hz}$, a large value of $\left|J_{\mathrm{AX}}-J_{\mathrm{Ax}}\right|$ indicates a large excess of one form. (Interchanging the values of $J_{\mathrm{Ax}}$ and $J_{\mathrm{Ax}}$ ' does not change the spectrum, and therefore nmr spectra cannot say which of $t t$ or $g g$ predominates. This must be decided by ir spectroscopy or potential energy calculations.) Doskocilova, et al ${ }^{33}$ estimated that in the syndiotacticlike isomer of 2,4,6-trichloroheptane, for values of $J_{\mathrm{Ax}}$ and $J_{\mathrm{Ax}}{ }^{\prime}$ of 11 and $2.5 \mathrm{~Hz}$, respectively, the relative populations of the $t t$ and $g g$ conformations of each $\mathrm{CH}_{3} \mathrm{CHClCH}_{2} \mathrm{CHClCH}_{2}$-dyad unit were 0.85 and 0.15 at $20^{\circ}$. Not unexpectedly, a comparable situation exists in $r$ dyads in the polymer, but the $t t$ form probably is even more highly favoured in the polymer since 
our values of 11 and $2 \mathrm{~Hz}$ for $J_{\mathrm{Ax}}$ and $J_{\mathrm{Ax}}$ were measured at $140^{\circ}$. As the temperature increases $\left(J_{\mathbf{A X}}-J_{\mathbf{A X}}\right)$ should decrease, as Doskocilova, et al., found. At $130^{\circ}$, they reported $J_{\mathrm{AX}}=10.2$ and $J_{\mathrm{AX}^{\prime}}=3.1 \mathrm{~Hz}$.

The solvents used in this work are moderately good solvents for polyvinyl chloride and a probable explanation for the increase in preference for the $t t$ conformation lies in the tendency for these solyents to extend the polymer chain more than if intramolecular steric interactions, as judged from model compounds, were the sole determining factor. It would be interesting to see if in poorer solvents a decrease in the end-to-end distance of the chain were reflected in the vicinal coupling constants.

The structure of polyvinyl chloride in solution is now well characterized. Both the configurational and conformational probabilities are known, and it should be possible to compute other properties such as the mean-square end-to-end distance. It has been suggested ${ }^{34}$ that in isotactic chains in solution the $3_{1}$ helix extends undisturbed for a considerable number of units. The only allowed helix reversal is of the type $(g t)(t g)$, the junction $(t g)(g t)$ being sterically unfavourable. Since we have shown that in polyvinyl chloride the $r$ sequences are almost as conformationally regular as $m$ sequences, then the majority of kinks in a polyvinyl chloride chain would occur at $m-r$ junctions of the form $(t g)(t t)$ and $(g t)(t t)$ or their mirror images.

\section{CHAIN DECOMPOSITION}

Finally, I should like to discuss briefly some work which has recently been carried out by R. Salovey ${ }^{35,37,38}$ and his coworkers in our laboratory on the dehydrochlorination of polyvinyl chloride initiated by high energy radiation, in this case $1 \mathrm{MeV}$ electrons from a van der Graaff accelerator. By this means, the formation of polyene structures may be initiated in a very clean and reproducible manner. By exercising careful temperature control, the process can be examined at various stages by uv spectroscopy. Thin films of polymer (ca. $4 \mathrm{~mm}$ ), free of stabilizers and moulded under vacuum, are irradiated at liquid nitrogen temperature $\left(-195^{\circ}\right)$ in vacuum. A single relatively small dose of 6-8 Mrad is applied in a period of the order of 2 minutes. This produces no optically observable effect provided the sample is maintained at $-195^{\circ}$. Upon warming the sample to $25^{\circ}$ but still maintaining it in vacuum, a multi-peaked spectrum begins to be observed (Figure 6), which increases steadily with time. This corresponds to the development of polyene sequences, which give rise to an intense colouration at later stages. It is curious and at present not entirely explained that as the intensity of this absorption increases, its form, at least down to $3000 \AA$ (the wavelength cut-off in earlier work ${ }^{35}$, carried out in Pyrex cells), remains unchanged, although it is known that for polyene structures of increasing length the uv absorption bands move to the $\operatorname{red}^{36}$. It is likely that beyond a certain length, the band structure converges to a long wavelength limit.

It is also probable that polyenyl radicals contribute to the uv absorption. Esr measurements (Figure 7) show clearly that free radicals are present and that they maintain a constant concentration for long periods of storage in vacuum while optical density increases (Figure 8 ). Thus, any proposed 
CONFIGURATION, CONFORMATION AND DEGRADATION OF PVC

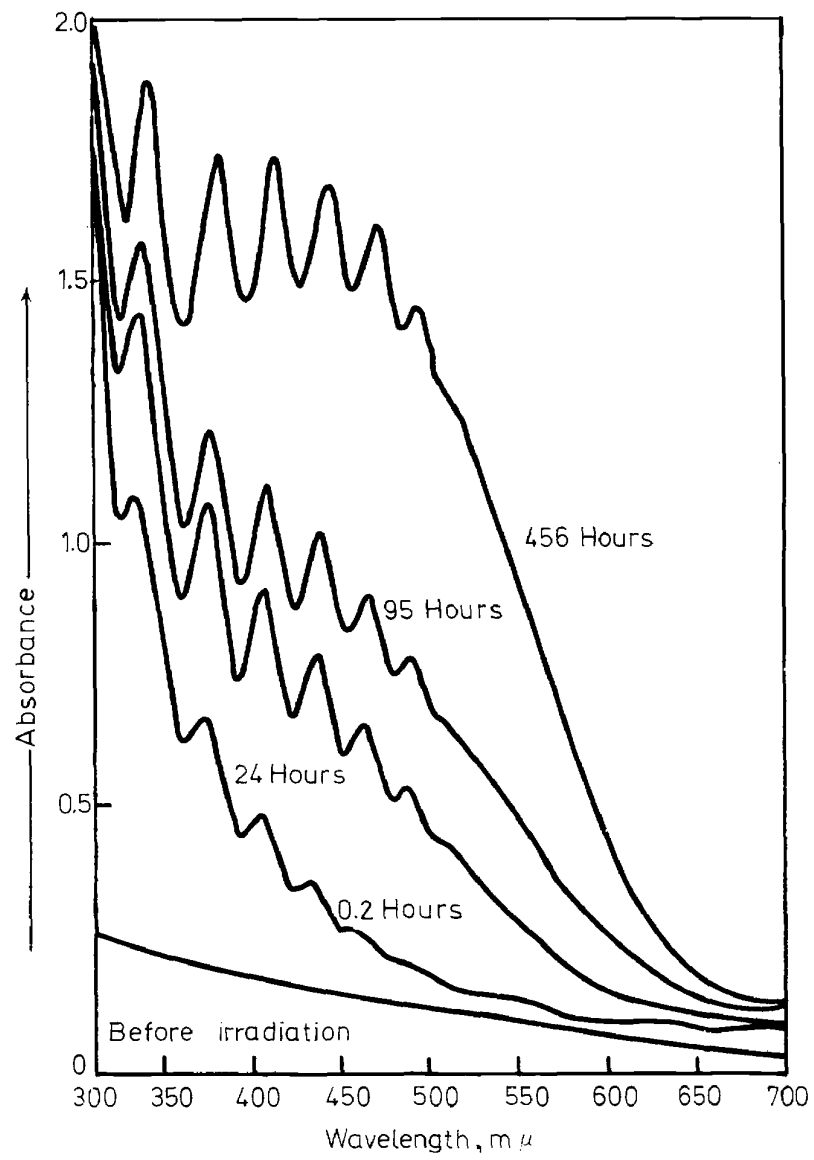

Figure 6. Absorption spectra of irradiated polyvinyl chloride following storage under vacuum (reference 35).

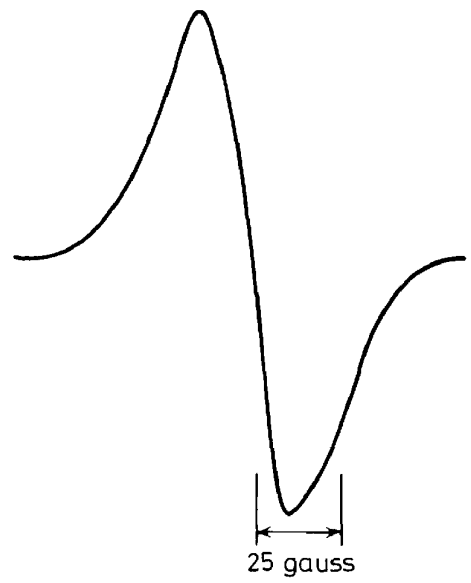

Figure 7. Electron spin resonance spectra of irradiated polyvinyl chloride (reference 35 ). 


\section{F. A. BOVEY}

mechanism for these degradative reactions under these conditions must involve conservation of radical species. The esr signal has the broad featureless appearance characteristic of polyenyl radicals, arising from a multitude of weak splittings owing to widespread electron delocalization.

The first steps in the degradation are probably the following:
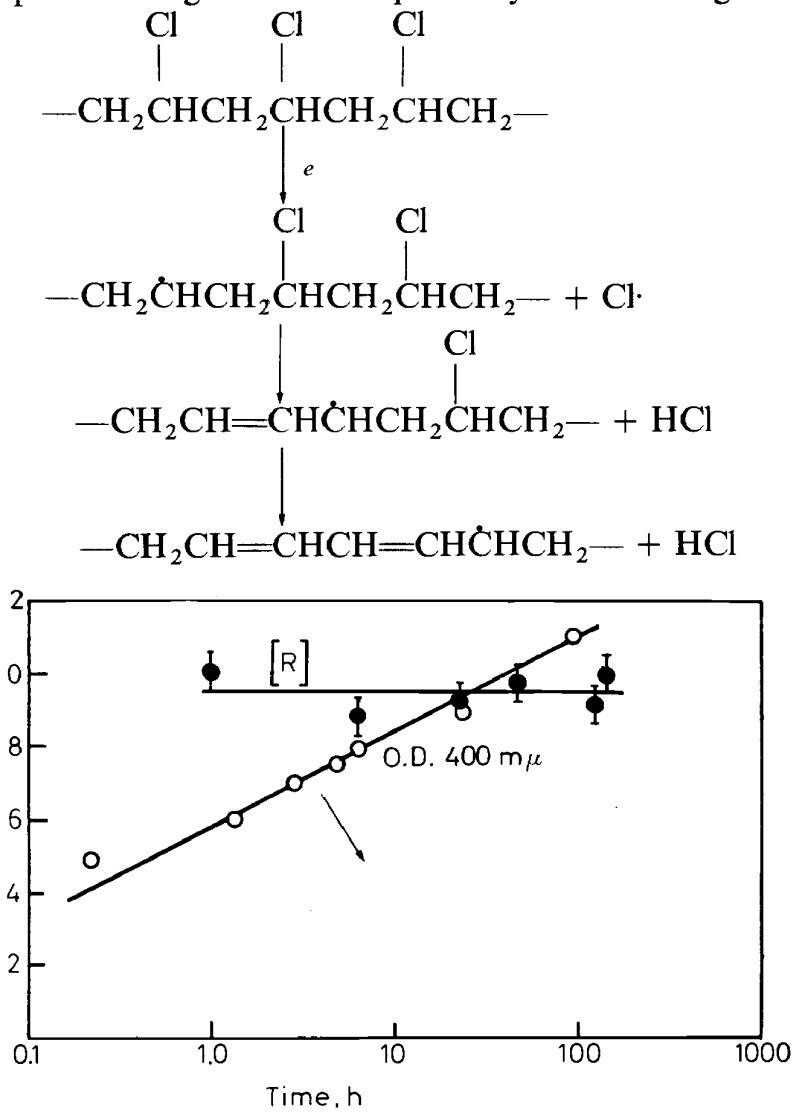

Figure 8. Optical density at $400 \mathrm{~m} \mu$ and relative concentration in irradiated polyvinyl chloride following storage under vacuum (reference 35 ).

In later studies using a specially designed cell ${ }^{37}$ and quartz optics ${ }^{37.38}$ spectral observations have been extended to $2000 \AA$. In Figure 9 are shown spectra of irradiated polyvinyl chloride obtained under these conditions. It is found (not shown in this figure) that there is an immediate large increase in absorbance near $2000 \AA$ after irradiation even when the storage temperature is maintained at $-120^{\circ}$. This is attributed to the alkyl radical, generated in reaction (1) above. Upon warming to $61^{\circ}$, there is a rapid development of an absorption peak at $2440 \AA$ which shifts to $2520 \AA$ as it grows in intensity. This is attributed to the allyl radical (reaction (2)). Upon continued storage, it passes through a maximum (in about $1 \mathrm{hr}$ at $25^{\circ}$; see Figure 10 ) and then decreases, while the polyene intensity increases at its expense. This corresponds to dehydrochlorination proceeding beyond reactions $(2,3)$. There is 
CONFIGURATION, CONFORMATION AND DEGRADATION OF PVC

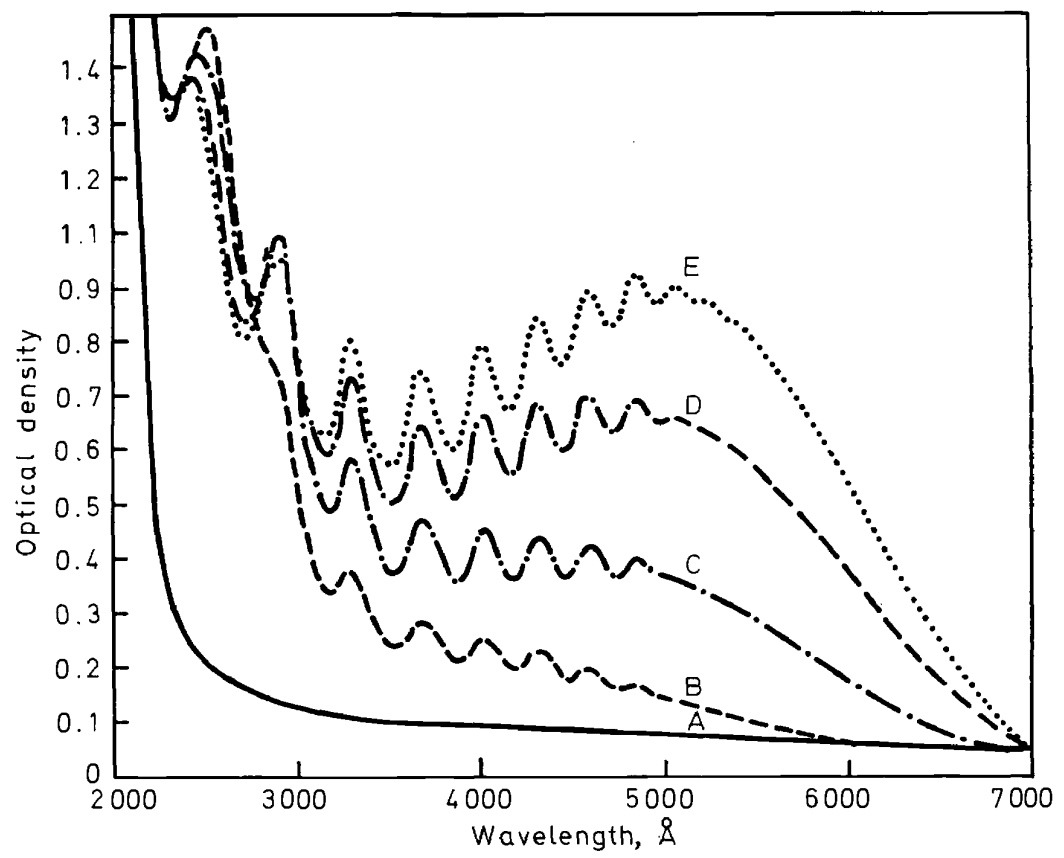

Figure 9. Polyene formation in irradiated polyvinyl chloride: A, control; B, irradiated to 5.9 Mrad, warmed to $61^{\circ}$; C, post-irradiation storage, $17.5 \mathrm{hr}$ at $61^{\circ} ; \mathrm{D}$, after warming to $100^{\circ}$; post-irradiation storage, $1.5 \mathrm{hr}$ at $90^{\circ}$ (reference 38 ).

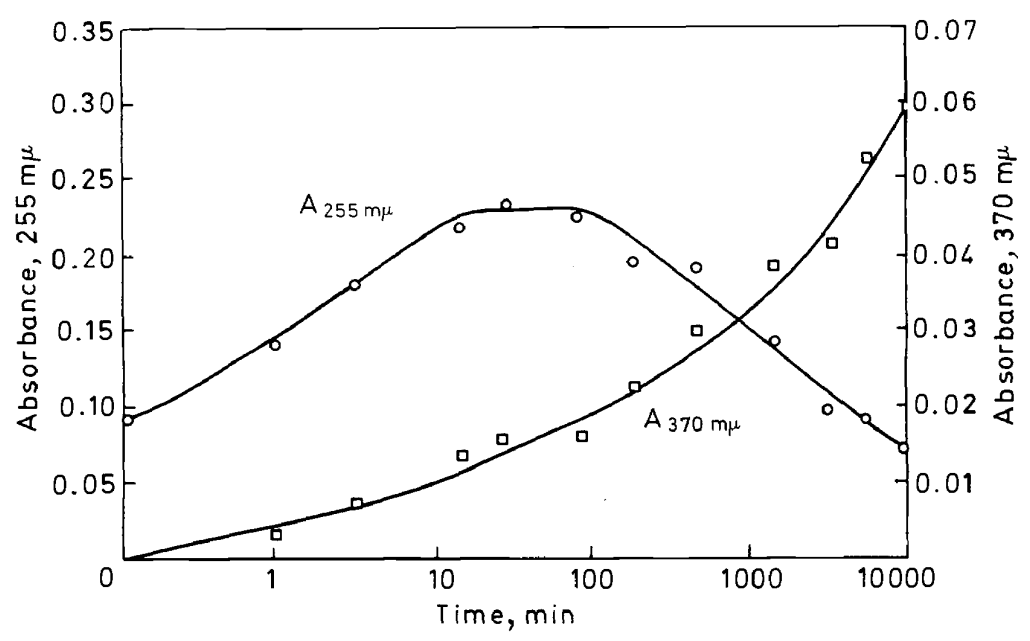

Figure 10. Optical density at 255 and $270 \mathrm{~m} \mu$ at $25^{\circ} \mathrm{C}$ of poly (vinyl chloride) irradiated at $-196^{\circ} \mathrm{C}$ and stored at $25^{\circ} \mathrm{C}$ in vacuum (reference 37 ). 


\section{F. A. BOVEY}

also a band at $2850 \AA$ (Figure 9) which behaves similarly. This is attributed to the dienyl radical produced in reaction (3).

The detailed spectral interpretation is actually somewhat complicated by the fact that the absorptions of the free radicals and the stable polyene structures overlap. Upon admission of oxygen to the stored irradiated films, the radicals are scavenged very rapidly but the polyenes are not seriously affected until after several hours of exposure. Differential absorption is observed at 2520,2910 and $3300 \AA$. The first of these corresponds to scavenging of the allyl radical, the others to the scavenging of the dienyl and trienyl radicals, respectively (Figure 11). Ultraviolet bands at 2580, 2850, 2230 and $3590 \AA$

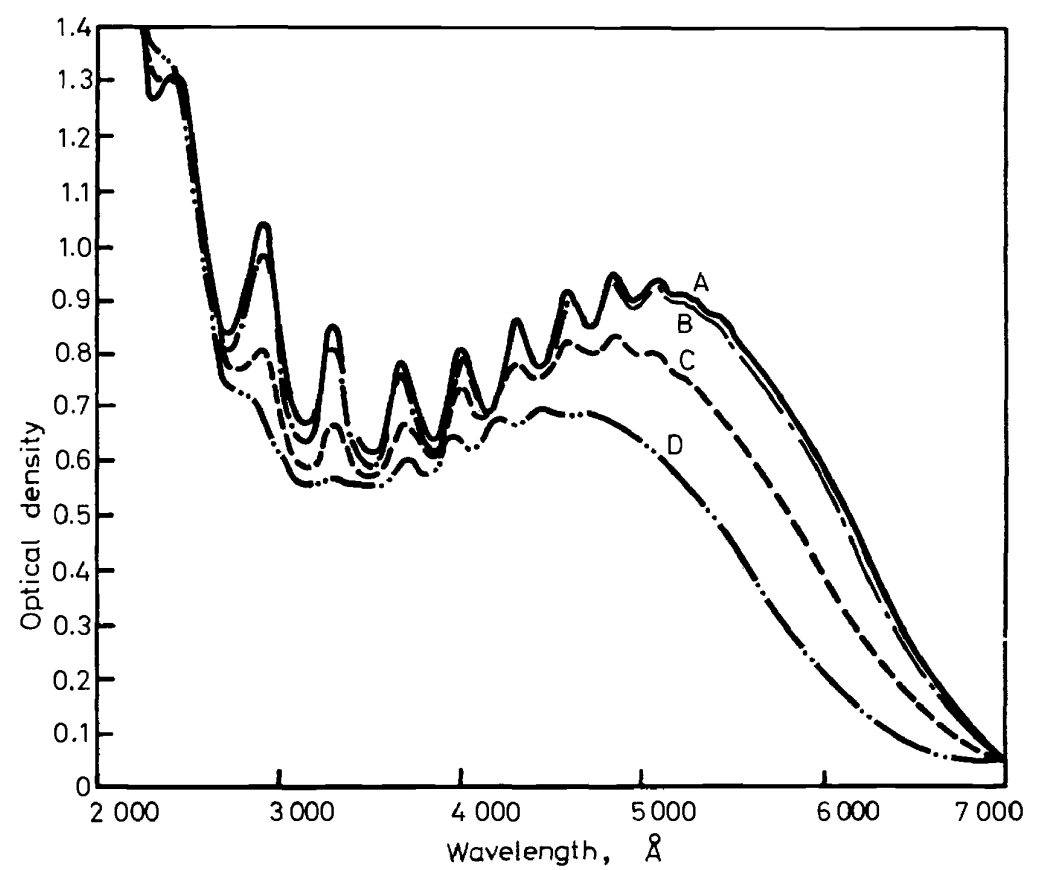

Figure 11. Effect of oxygen on irradiated polyvinyl chloride: A, film of Figure 9, additional $65 \mathrm{hr}$ storage at $25^{\circ} ; \mathrm{B}, 1 \mathrm{~min}$ exposure to oxygen at $25^{\circ} ; \mathrm{C}, 4 \mathrm{hr}$ exposure to oxygen at $25^{\circ} ; \mathrm{D}, 23 \mathrm{hr}$ exposure to oxygen at $25^{\circ}$ (reference 38 ).

have been assigned to allyl, dienyl, trienyl and tetraenyl free radicals, respectively, in irradiated polyethylene ${ }^{39}$. The large absorbance remaining in the 2000-3000 $\AA$ region even after several hours of exposure to oxygen, and the concomitant disappearance of the banded structure at longer wavelength, are attributed to development of a variety of oxidation products absorbing at a variety of wavelengths.

There thus appears to be direct evidence for the reactions proposed above, or at least for very similar reactions. It is possible that the dehydrochlorination may also proceed in part by initial removal of $\alpha$ or $\beta$ protons under 
irradiation. Chain transfer and recombination of radicals to produce crosslinks undoubtedly also occur.

The relevance of these studies to the reactions occurring in the thermal degradation of polyvinyl chloride may perhaps be questioned. We suggest that the two processes probably have much in common, but that they are by no means identical. For example, typical radical scavengers inhibit the development of colour under irradiation but do not have an equal effect on thermal discolouration. Whatever the truth may prove to be, we are nevertheless directly concerned with the degradation occurring under high energy radiation, for the radiation crosslinking of polyvinyl chloride is under development in our laboratories for commercial use in the production of wire insulation.

\section{REFERENCES}

D. Doskocilova. J. Polymer Sci.2, 421 (1964).

D. Doskocilova and B. Schneider. Collection Czech. Chem. Commun. 29, 2290 (1964).

3 S. Satoh. J. Polymer Sci. A2, 5221 (1964).

4 H. G. Clark. Makromol. Chem. 63, 69 (1963).

5. T. Shimanouchi, M. Tasumi and Y. Abe. Makromol. Chem. 86, 43 (1965).

6 Y. Abe, M. Tasumi, T. Shimanouchi, S. Satoh and R. Chujo. J. Polymer Sci.4A-1. 1413 (1966).

7 D. Doskocilova, J. Stokr, B. Schneider, H. Pivcova, M. Kolinsky, J. Petranek and D. Lim. J. Polymer Sci. C16, 215 (1967).

8 U. Johnsen. J. Polymer Sci. 54, 56 (1961).

9 F. A. Bovey and G. V. D. Tiers. Chem. Ind. London (1962) 1826.

10 R. Chujo, S. Satoh, T. Ozeki and E. Nagai. J. Polymer Sci. 61, S12 (1962).

11 W. C. Tincher. J. Polymer Sci. 62, S148 (1962).

12 F. A. Bovey, E. W. Anderson, D. C. Douglass and J. A. Manson. J. Chem. Phys. 39, 1199 (1963).

13 S. Satoh. J. Polymer Sci. A2, 5221 (1964).

14 W. C. Tincher. Makromol. Chem. 85, 20 (1965).

15 T. Yoshino and J. Komiyama. J. Polymer Sci. B3, 311 (1965).

16 O. C. Böckman. J. Polymer Sci. A3, 3399 (1965).

17 B. Schneider, J. Stokr, D. Doskocilova, M. Kolinsky, S. Sykora and D. Lim. IUPAC Symp. Macromol. Chem., Prague, 1965, preprints, p. 599.

18 J. Bargon, K. H. Hellwege and U. Johnsen. Makromol. Chem. 95, 187 (1966).

19 K. C. Ramey. J. Phys. Chem. 70, 25 (1966).

20 S. Enemoto, M. Asahina and S. Satoh. J. Polymer Sci. 4Ä-1, 1373 (1966).

21 F. A. Bovey, F. P. Hood, E. W. Anderson and R. L. Kornegay. J. Phys. Chem. 71, 312 (1967).

22 U. Johnsen and K. Kolbe. Kolloid-Z. 221, 64 (1967).

${ }^{23}$ F. Heatley and F. A. Bovey. Macromolecules 2241 (1969).

${ }^{24}$ L. Cavalli, G. C. Borsini, G. Carraro and G. Confalonieri. J. Polymer Sci. 8A-1, 801 (1970).

25 F. A. Bovey. J. Polymer Sci. 4659 (1960).

26 J. W. Fordham, P. H. Burleigh and C. L. Sturm. J. Polymer Sci. 4659 (1960).

27 (a) H. Germar, K. H. Hellwege and U. Johnsen, Makromol. Chem. 60106 (1963).

27 (b) G. Talamini and G. Vidotto. Polymer Previe us 2227 (1966).

28 J. D. Cotman, Jr. Ann. N.Y. Acad. Sci. 57417 (1953).

29 M. H. George, R. J. Grisenthwaite and R. F. Hunter. Chem. Ind. (London) 1114.(1958).

${ }^{30}$ G. Boccato, A. Rigo, G. Talamini and F. Zilio-Grandi. Makromol. Chem. 108218 (1967).

${ }^{31}$ I. Rosen, P. H. Burleigh and J. F. Gillespie. J. Polymer Sci. 5431 (1961).

32 B. Schneider, J. Stokr, D. Doskocilova, S. Sykora, J. Jakes and M. Kolinsky. J. Polymer Sci. 22-C, 1073 (1969).

${ }^{33}$ D. Doskocilova, J. Stokr, B. Schneider, H. Pivcova, M. Kolinsky, J. Petranek and D. Lim. J. Polymer Sci. 16-C, 214 (1967).

34 P. J. Flory, J. E. Mark and A. Abe. J. Am. Chem. Soc. 88, 639 (1966).

35 R. Salovey, J. P. Luongo and W. A. Yager. Macromolecules 3198 (1969). 


\section{F. A. BOVEY}

${ }^{36}$ F. Sondheimer, D. A. Ben-Efraim and R. Wolovsky. J. Am. Chem. Soc. 831675 (1961).

37 R. Salovey and J. P. Luongo. J. Polymer Sci. 8A-1 209 (1970).

38 R. Salovey, R. V. Albarino and J. P. Luongo. Macromolecules 3314 (1970).

39 D. M. Bodily and M. Dole. J. Chem. Phys. 451428 (1966). 